\title{
Evidence for a co-moving sub-stellar companion of GQ Lup ${ }^{\star}$
}

\author{
R. Neuhäuser ${ }^{1}$, E. W. Guenther ${ }^{2}$, G. Wuchterl ${ }^{1}$, M. Mugrauer ${ }^{1}$, A. Bedalov ${ }^{1}$, and P. H. Hauschildt ${ }^{3}$ \\ 1 Astrophysikalisches Institut, Universität Jena, Schillergäßchen 2-3, 07745 Jena, Germany \\ e-mail: rne@astro.uni-jena.de \\ 2 Thüringer Landessternwarte Tautenburg, Sternwarte 5, 07778 Tautenburg, Germany \\ 3 Hamburger Sternwarte, Gojenbergsweg 112, 21029 Hamburg, Germany
}

Received 6 October 2004 / Accepted 27 March 2005

\begin{abstract}
We present a companion of the $\leq 2 \mathrm{Myr}$ young classical T Tauri star GQ Lup in the Lupus star forming region at $140 \pm 50 \mathrm{pc}$ from imaging, astrometry, and spectroscopy. With direct $K$-band imaging using VLT/NACO, we detected an object 6 mag fainter than GQ Lup located 0.7" west of it. Compared to images obtained 2 to 5 years earlier with Subaru/CIAO and HST/PC, this object shares the proper motion of GQ Lup by 5 and $7 \sigma$, respectively, hence it is a co-moving companion. Its $K-L^{\prime}$ color is consistent with a spectral type early to mid L. Our NACO $K$-band spectrum yields spectral type M9- 44 with $\mathrm{H}_{2} \mathrm{O}$ and $\mathrm{CO}$ absorption, consistent with the new GAIA-Dusty template spectrum for $\log g \simeq 2$ to 3 and $T_{\text {eff }} \simeq 2000 \mathrm{~K}$ with $\sim 2 R_{\text {jup }}$ radius at $\sim 140 \mathrm{pc}$, hence few Jupiter masses. Using the theoretical models from Wuchterl \& Tscharnuter (2003), Burrows et al. (1997), and Baraffe et al. (2002), the mass lies between 1 and 42 Jupiter masses.
\end{abstract}

Key words. low-mass stars - substellar companions - brown dwarfs - extra-solar planets - GQ Lup

\section{Introduction: Direct detection of planets}

Direct detection of sub-stellar companions is difficult because of the large dynamic range between the faint companion and the close-by, much brighter star. Due to contraction, Myr young sub-stellar objects are brighter than Gyr older ones. Hence, young nearby stars would be the best targets for the direct detection of sub-stellar companions. The mass of a detected substellar companion can be estimated from the observed companion magnitude and the assumed or known age and distance of the primary star, using theoretical model calculations. Chauvin et al. (2004) reported the detection of a faint object near the brown dwarf 2M1207, a potential member of TWA at $~ 8 \mathrm{Myr}$, whose infrared (IR) color and $H$-band spectrum are consistent with an L dwarf, hence $\sim 5 M_{\text {jup }}$ (Baraffe et al. 2002 models), if bound (astrometry missing).

Here, we present evidence for a sub-stellar companion around GQ Lup located in the Lupus I cloud (Tachihara et al. 1996), a young K7eV-type classical T Tauri star with low extinction, but with mid- and far-IR excess (Hughes et al. 1994), i.e. a disk, and also both soft and hard X-ray emission (Krautter et al. 1997), rare for classical T Tauri stars (Neuhäuser et al. 1995), so that we included this star in our planet search programs by radial velocity and direct imaging since March 1999. As distance towards GQ Lup in the Lupus I cloud, we use

^ Based on observations obtained on Cerro Paranal, Chile, in ESO programs 73.C-0164 and 273.C-5047 as well as on data collected at the Subaru Telescope and the Hubble Space Telescope, both obtained from their science archives.
$140 \pm 50$ pc (Wichmann et al. 1998; Neuhäuser \& Brandner 1998; Knude \& Hog 1998), the age of GQ Lup is 0.1 to $2 \mathrm{Myr}$, depending on the set of models used (we work with $1 \pm 1 \mathrm{Myr}$ ).

\section{Direct observations of a wide companion}

\subsection{AO imaging detection and photometry}

We observed GQ Lup with Yepun (ESO-VLT-UT4) on Cerro Paranal, Chile, using the Adaptive Optics (AO) instrument NACO (Naos-Conica, Rousset et al. 2003) on 25 June 2004 in visitor mode using the S13 camera $\left(14^{\prime \prime} \times 14^{\prime \prime}\right.$ field $)$ in the $K_{\mathrm{s}}$-band. We took 200 co-adds $\times 0.347 \mathrm{~s} \times 27$ jitter positions. Data reduction was done with eclipse: dark subtraction, flat devision, shift+add. A companion candidate is found $0.7^{\prime \prime}$ west of GQ Lup (Fig. 1). By comparison with the primary $(K=7.096 \pm 0.020 \mathrm{mag}, 2 \mathrm{MASS})$, we got $K_{\mathrm{s}}=13.1 \pm 0.1 \mathrm{mag}$ for the companion candidate with aperture photometry after subtracting the primary.

$K$ - and $L^{\prime}$-band images of GQ Lup were obtained with the Subaru Coronagraphic Imager with Adaptive Optics (CIAO, Murakawa et al. 2004), in program o02312, retrieved by us from the public Subaru archive SMOKA. We reduced the data in the above way. The candidate is detected in both $K$ (as with NACO) and $L^{\prime}$, where we obtain $L^{\prime}=11.7 \pm 0.3 \mathrm{mag}$ by comparing the companion candidate with the primary ${ }^{1}$.

${ }^{1} L=6.05 \pm 0.13 \mathrm{mag}$ from Glass \& Penston (1974) and Hughes et al. (1994); hence, $L^{\prime} \simeq 6.05 \mathrm{mag}$; the difference between $L$ and $L^{\prime}$ for $\mathrm{K} 7$ is marginal, within the 0.3 mag error given of the companion. 


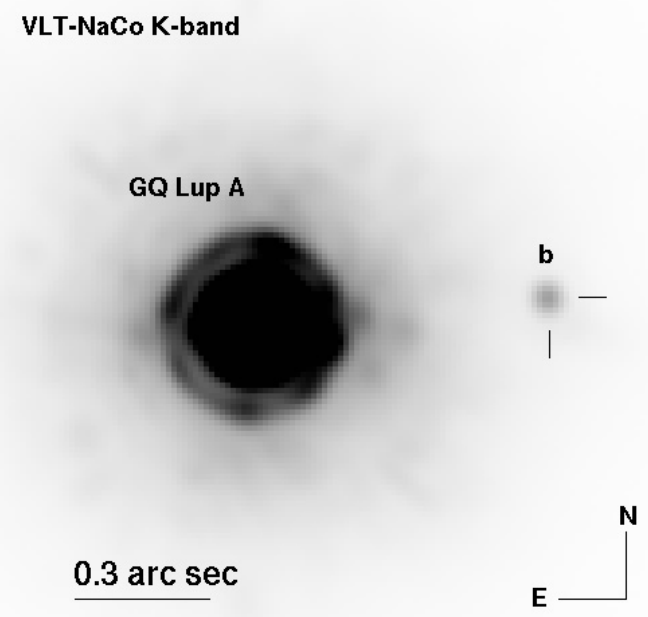

Fig. 1. VLT-NACO $K_{\mathrm{s}}$-band image of GQ Lup and its 6 mag fainter companion candidate $0.7325 \pm 0.0034^{\prime \prime}$ west.

The primary has $A_{V}=0.4 \pm 0.2$ mag (Batalha et al. 2001), hence $A_{K}=0.04 \pm 0.02$ and $A_{L^{\prime}} \simeq 0.02 \pm 0.01$ mag (Rieke \& Lebofsky 1985). Applying this extinction to the companion, we derive $\left(K-L^{\prime}\right)_{0} \simeq 1.4 \pm 0.3 \mathrm{mag}$, consistent with L2-7 (Stephens et al. 2001; Golimowski et al. 2004) ${ }^{2}$. At $\sim 140$ pc, both the primary and the companion candidate are by $\sim 3$ mag brighter than main-sequence K7V stars and Gyr old (main-sequence) early to mid L-dwarfs as far as the absolute magnitudes are concerned, consistent with both being young.

GQ Lup and its companion candidate are also detected in archived images obtained with the Hubble Space Telescope (HST) Wide Field Planetary Camera No. 2 (WFPC2) in program SNAP 7387 (retrieved by us from the public MAST archive at STScI) in the filters $F 606 W$ and $F 814 W$. Data reduction was performed for the HST data as in Neuhäuser et al. (2002). Since the companion candidate is detected only marginally, we cannot obtain reliable optical colors.

\subsection{Astrometry}

To study whether primary and companion candidate form a common proper motion pair, we compare separations and position angles PA at the different epochs. We use $21.33 \pm$ $0.02 \mathrm{mas} /$ pixel as CIAO pixel scale (Fukagawa et al. 2003, 2004) and $45.545 \pm 0.005$ mas/pixel for HST/PC (Holtzman et al. 1995). The positions of primary and companion are determined by Gaussian centering, after primary PSF subtraction as far as the companion is concerned, and the errors given include the pixel scale error. The separations measured are given in Table 1 ( $1 \sigma$ errors) including the aquisition images for the spectroscopy, obtained with the NACO S27 camera with $27.07 \pm 0.11 \mathrm{mas} /$ pixel. The astrometric precision is best in our own deep NACO image (June 2004) obtained with the S13 camera with $13.23 \pm 0.05$ mas/pixel. Scale and orientation of S13 and S27 were obtained by us using $\sigma$ Ori AD

2 The primary has a $K$-band excess due to its disk of $\sim 2 \mathrm{mag}$ (from a K7V blackdody fit to its $U B V R I J H K$ mags).

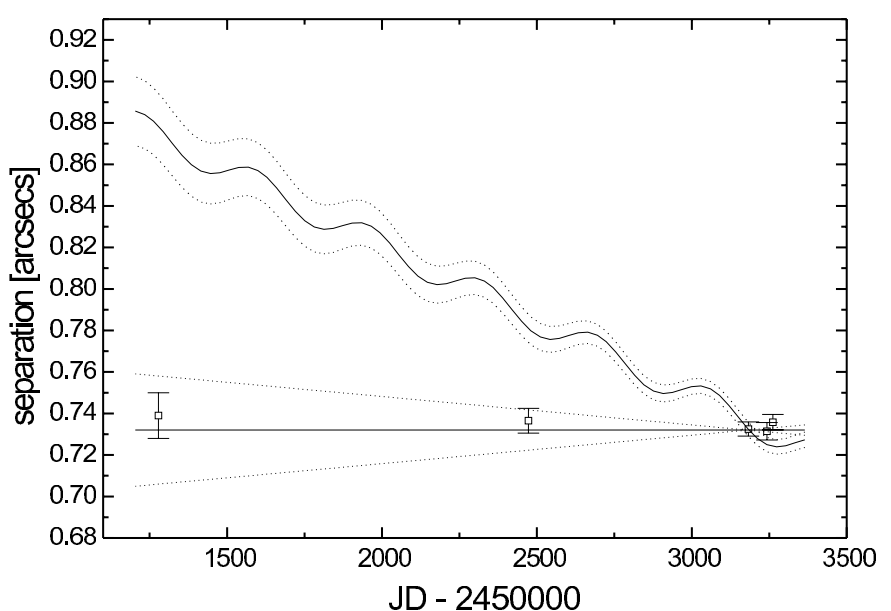

Fig. 2. Observed separation between primary star GQ Lup A and companion candidate with HST/PC (left), Subaru/CIAO (middle) and VLT/NACO (right, first the June 2004 image, then the Aug. and Sep. 2004 aquisition images, all consistent within $1 \sigma$ ) compared to the expectation when bound (no change in separation as straight full line $\pm 5 \mathrm{mas} / \mathrm{yr}$ for orbital motion as straight dotted lines) or when background (motion of GQ Lup A alone to the SW due to its proper motion \pm its error with wobble due to expected parallactic motion of 7.1 mas). We can reject the background hypothesis by $4.9 \sigma$ (NACO-CIAO) plus $7.2 \sigma$ (NACO-HST).

Table 1. Observing log, $F W H M$, and separations.

\begin{tabular}{llrc}
\hline \hline Tel./Instr. & Obs. Date & $F W H M$ & \multicolumn{1}{c}{ Separation } \\
\hline HST/PC & 10 Apr. 1999 & $95 \mathrm{mas}$ & $739 \pm 11 \mathrm{mas}$ \\
Subaru/CIAO & 17 Jul. 2002 & $150 \mathrm{mas}$ & $736.5 \pm 5.7 \mathrm{mas}$ \\
VLT/NACO & 25 Jun. 2004 & $68 \mathrm{mas}$ & $732.5 \pm 3.4 \mathrm{mas}$ \\
VLT/NACO & 25 Aug. 2004 & $74 \mathrm{mas}$ & $731.4 \pm 4.2 \mathrm{mas}$ \\
VLT/NACO & 14 Sep. 2004 & $72 \mathrm{mas}$ & $735.8 \pm 3.7 \mathrm{mas}$ \\
\hline
\end{tabular}

(Hipparcos: $12.980^{\prime \prime}$ at $84.2^{\circ}$ ) and GJ $852 \mathrm{AB}$ taking into account the orbital motion within them. The position angle of the detector was tilted by $0.14 \pm 0.25^{\circ}$ to the east.

If the faint object would be a background object, it would have negligible proper motion and we would see a change in separation due to the proper and parallactic motion of GQ Lup A (Fig. 2). Its proper motion is $\mu_{\alpha}=-27 \pm 3$ mas $/ \mathrm{yr}$ and $\mu_{\delta}=-14 \pm 3 \mathrm{mas} / \mathrm{yr}$ (Teixeira et al. 2000, Camargo et al. 2003) at $140 \pm 50 \mathrm{pc}$ distance. The background hypothesis can be rejected by 4.9 and $7.2 \sigma$ by comparing NACO with CIAO and HST, respectively. The PA of the companion is $275.45 \pm 0.30^{\circ}$ in the NACO image in June 2004 (detector orientation calibrated); if the candidate would be a non-moving background object, the PA should have been $269.89 \pm 1.03^{\circ}$ at the time of the HST epoch, but we observe $275.62 \pm 0.86^{\circ}$ (detector orientation from fits file header, known to be stable and precise), i.e. $4.3 \sigma$ deviant from the background hypothesis. Hence, the faint object is co-moving with GQ Lup A. The probability to find by chance a fore- or background $\mathrm{M}$ or L dwarf with the same proper motion near GQ Lup A is negligible. 


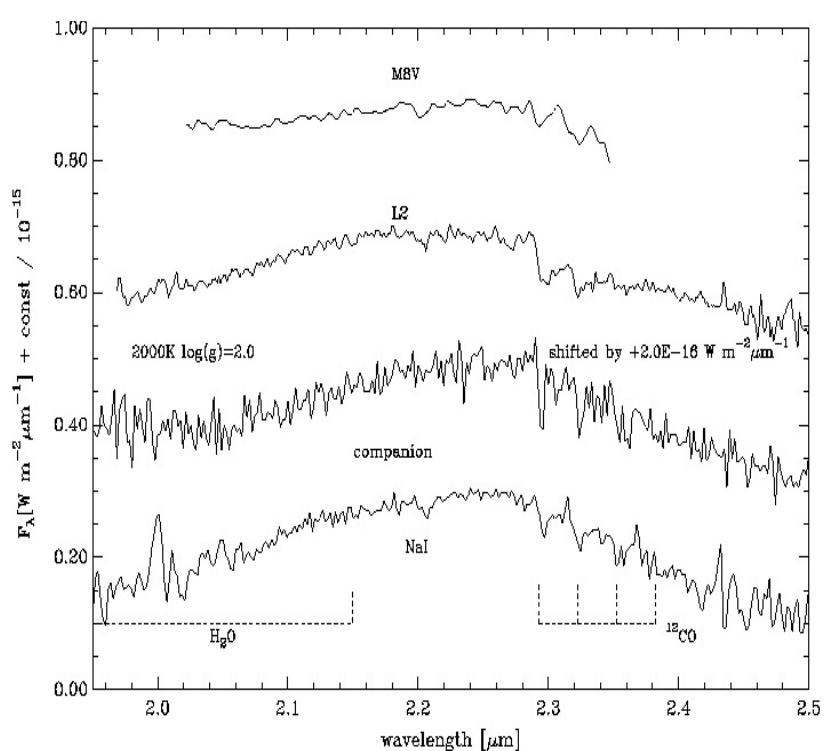

Fig. 3. Our flux-calibrated $K$-band NACO spectrum of the companion of GQ Lup (bottom) compared to the young M8 brown dwarfs in Cha I (top) from Comerón et al. (2000), L2 (2MASSW J0829066+145622, 2nd from top) from Reid et al. (2001), and a GAIA-Dusty template spectrum for $2000 \mathrm{~K}$ and $\log g=2$, which compares well with the companion.

\subsection{Spectroscopy of the companion}

To check whether the companion is indeed cool, we obtained a $K$-band spectrum with VLT/NACO using the S54-4-SK mode from 1.79 to $2.57 \mu \mathrm{m}$ with resolution 700 and nodding along the 172 mas slit. Two spectra were obtained on 25 Aug. and 14 Sep. 2004 with 20 and 40 spectra, respectively, exposed for $30 \mathrm{~s}$ each and reduced in the normal way: dark subtraction, flat devision, shift+add, and wavelength calibration (Fig. 3).

We confirmed that the faint object was positioned well in the center of the slit in every individual spectrum. Wavelength dependant refraction and Strehl-ratios through the narrow slit can change the slope of the spectrum: The light-loss due to refraction, following Schubert \& Walterscheid (2000) with the given airmasses, parallactic and positional angle, is 1.5 and $1.6 \%$ at the 1.9 and $2.5 \mu \mathrm{m}$, respectively, compared to the flux at $2.35 \mu \mathrm{m}$. Given the seeing and slit-width, the slit efficiency is $76.4 \%$ at $1.67 \mu \mathrm{m}$ and $79.0 \%$ at $2.18 \mu \mathrm{m}$, so that the flux in the blue is reduced by $2.5 \%$. In total, $2.0 \%$ of the flux is missing in the blue compared to the middle of the spectrum and only $0.8 \%$ in the red. The slit-loss is partly compensated by higher Strehl-ratio, because the companion flux peaks in the middle of the $K$-band.

The flux of the companion is calibrated once with the spectrum of the $\mathrm{K} 7 \mathrm{eV}$ primary star GQ Lup A observed simultaneous and once with the telluric standard HD 159402 (B3III), observed in the same night. The large telluric absorption features below $2.06 \mu \mathrm{m}$ and above $2.42 \mu \mathrm{m}$ nevertheless cause a considerable amount of noise in this part of the spectrum. The Reid et al. (2001) K1 spectral index is 0.13 to 0.39 giving M9-L3. The McLean et al. (2003) $\mathrm{H}_{2} \mathrm{O}-\mathrm{D}$ ratio is 0.67 to 0.89 indicating L2-7 (cf. Cushing et al. 2005). The NaI doublet has $W_{\lambda}=4.0 \pm 0.5 \AA$ for the primary, but $\leq 2 \AA$ for the companion (possibly partly telluric), which implies a spectral type $\sim$ M9 or later (Cushing et al. 2005; Comerón et al. 2000) or $\sim$ L2 or later (Gorlova et al. 2003); even for strongly reddened M stars, the NaI line should still be strong (Greene \& Lada 1996). The CO band head at $2.295 \mu \mathrm{m}$ is also present in M-dwarfs, increases slightly in strength for early L, but weakens again in later types. This band head is clearly visible with CO-index $\sim 0.86$, i.e. M6 to L0 (Gorlova et al. 2003). The average of all the above estimates is L1.5 \pm 2.5 , or M9 to L4. This is consistent with the dereddened $K-L^{\prime}$ color; hence no evidence for additional extinction due to, e.g., a disk around the companion.

\section{Mass determination and discussion}

Our companion, bound to a $1 \pm 1$ Myr young star, may be the youngest and lowest-mass companion ever imaged. Hence, it is difficult to compare it to field (old) L-dwarfs. From the $K$-band CO-index being $0.862 \pm 0.035$ we obtain the gravity $\log g=2.52 \pm 0.77$, a slight extrapolation from late-M to early-L (Gorlova et al. 2003), hence uncertain. Since neither spectral type nor gravity are well constrained, the temperature $T_{\text {eff }}$ is only weakly constrained to $\sim 1600$ to $2500 \mathrm{~K}$ (or $2050 \pm 450 \mathrm{~K}$ ) from Gorlova et al. (2003) and references therein as well as Reid et al. (1999), Stephens et al. (2001), Burgasser et al. (2002), Nakajima et al. (2004), and Golimowski et al. (2004).

We compared our observed spectrum with the theoretical template spectra from the GAIA-Dusty model (Brott \& Hauschildt, in prep.), updated from Allard et al. (2001), with improved molecular dissociation constants, more dust species with opacities, spherical symmetry, and a mixing length parameter $1.5 \cdot H_{\mathrm{p}}$; we tried $T_{\text {eff }}=2000$ and $2900 \mathrm{~K}$ and $\log g=0,2$, and 4. A good fit is obtained only for $2000 \mathrm{~K}$ and $\log g=2$ (Fig. 3). Because the fit for $\log g=0$ is much worse than for $\log g=4$, where the continuum at 2.22 to $2.3 \mu \mathrm{m}$ and the depth of the $\mathrm{CO}$ lines are not reproduced, and because of the $\log g$ from the CO-index (see above), we conclude log $g \simeq 2$ to 3 . The observed flux can be reproduced for an object with $\sim 2 R_{\text {jup }}$ radius at $140 \mathrm{pc}$.

With B.C.K $=3.3 \pm 0.1 \mathrm{mag}$ for M9-L4 and $K-L^{\prime} \simeq$ $1.4 \pm 0.3$ (Golimowski et al. 2004), $K_{\mathrm{s}}=13.1 \pm 0.2 \mathrm{mag}$ for the companion, and $140 \pm 50 \mathrm{pc}$ distance, the luminosity is $\log \left(L / L_{\odot}\right)=-2.37 \pm 0.41$ for the companion. We can now plot it into an H-R diagram to compare with models. From Burrows et al. (1997) Fig. 7, the companion has $\sim 12$ to $32 M_{\text {jup }}$ (from $L$ and age), but only $\sim 3$ to $9 M_{\text {jup }}$ from Figs. 9 and 10 (from $\mathrm{T}$ and age). It is similar in Baraffe et al. (2002) Fig. 2: the companion has $\sim 3$ to $16 M_{\text {jup }}$ from $T$ and age, but $\sim 11$ to $42 M_{\text {jup }}$ from L and age; $\sim 12$ to $42 M_{\text {jup }}$ from $M_{\mathrm{K}}$ and age (see also perso.ens-lyon. fr/isabelle.baraffe). Burrows et al. and Baraffe et al. start with an assumed internal structure without collapse, so that their models are uncertain up to a few Myrs (Baraffe et al. 2002). 


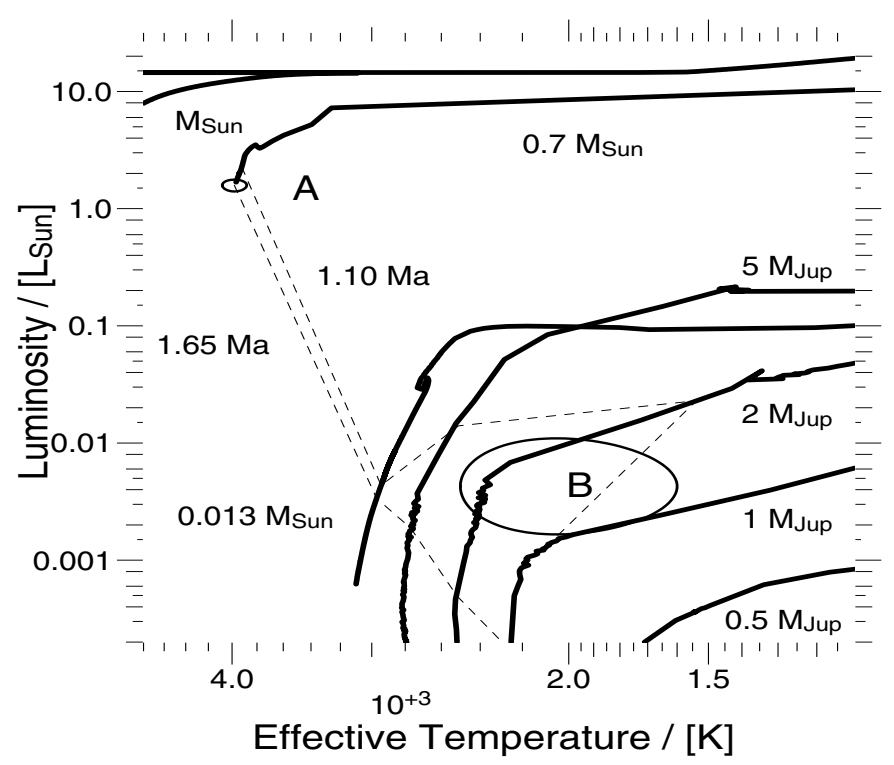

Fig. 4. H-R diagram with tracks from Wuchterl \& Tscharnuter (2003) plus more tracks calculated by us (from top to bottom) for 1 and $0.7 M_{\odot}$ as well as $13,5,2,1$, and $0.5 M_{\text {jup }}$. The isochrones (dashed) for 1.10 and $1.65 \mathrm{Myr}$ connect the end of the $0.7 M_{\odot} \operatorname{track}$ (star A) with the planetary tracks. Components $\mathrm{A}$ and $\mathrm{B}$ ( $1 \sigma$ errors) are co-eval at $\sim 1.1 \mathrm{Ma}$.

Wuchterl \& Tscharnuter (2003) include the initial collapse (Fig. 4): tracks for masses of 1 to $0.013 M_{\odot}$ are radiation fluid-dynamical calculations of the collapse of initially marginally unstable Bonnor-Ebert-spheres; planetary tracks are models in the framework of the nucleated instability hypothesis (Wuchterl et al. 2000; Wuchterl, in preparation). All ages are counted from first photosphere formation. The age-offset visible on the $1.10 \mathrm{Myr}$ isochrone, at the transition from the $0.013 M_{\odot}$ brown dwarf collapse to the $5 M_{\text {jup }}$ planetary track is due to the time-offset caused by the planetary core formation ( $\leq 10^{5} \mathrm{yr}$ ). The companion has a mass of $\sim 1$ to $2 M_{\text {jup }}(1 \sigma)$ and is co-eval with the star ( 1.1 Myr). From this model (Fig. 4$)$, we expect $\log g \simeq 2.4$ and $\sim 1.8 R_{\text {jup }}$ radius, consistent with our spectrum and the best fitting GAIA-Dusty model spectrum (Fig. 3).

The most critical point in the mass determination of the companion (candidates) of GQ Lup and 2M1207 are the models, which may be off by an unknown factor for low ages (few Myr); they need to be calibrated, before the mass of such companions can be determined confidently.

Acknowledgements. We thank N. Reid for his IR spectra of L-dwarfs, an anonymous referee for very good suggestions, and the ESO USG and Paranal teams for perfect support. R.N. would like to thank D.L.N. for long-term support.

\section{References}

Allard, F., Hauschildt, P. H., Alexander, D. R., Tamanai, A., \& Schweitzer, A. 2001, ApJ, 556, 357

Baraffe, I., Chabrier, G., Allard, F., \& Hauschildt, P. H. 2002, A\&A, 382,563

Burgasser, A. J., Kirkpatrick, J. D., Brown, M. E., et al. 2002, ApJ, 564,421

Burrows, A., Marley, M., Hubbard, W., et al. 1997, ApJ, 491, 856

Camargo, J. I. B., Ducourant, C., Teixeira, R., et al. 2003, A\&A, 409, 361

Chauvin, G., Lagrange, A., Dumas, C., et al. 2004, A\&A, 425, L29

Comerón, F., Neuhäuser, R., \& Kaas, A. A. 2000, A\&A, 359, 269

Cushing, M. C., Rayner, J. T., \& Vacca, W. D. 2005, ApJ, in press [arXiv: astro-ph/0412313]

Fukagawa, M., Tamura, M., Itoh, Y., Hayashi, S. S., \& Oasa, Y. 2003, ApJ, 590, L49

Fukagawa, M., Hayashi, M., Tamura, M., et al. 2004, ApJ, 605, L53

Glass, I. S., \& Penston, M. V. 1974, MNRAS, 167, 237

Golimowski, D., Leggett, S., Marley, M., et al. 2004, AJ, 127, 3516

Gorlova, N. I., Meyer, M. R., Rieke, G. H., \& Liebert, J. 2003, ApJ, 593, 1074

Greene, T. P., \& Lada, C. J. 1996, AJ, 112, 2184

Holtzman, J. A., Hester, J. J., \& Casertano, S. 1995, PASP, 107, 156

Hughes, J., Hartigan, P., Krautter, J., \& Kelemen, J. 1994, AJ, 108, 1071

Knude, J., \& Hog, E. 1998, A\&A, 338, 897

Krautter, J., Wichmann, R., Schmitt, J. H. M. M., et al. 1997, A\&AS, 123,329

McLean, I. S., McGovern, M. R., Burgasser, A. J., et al. 2003, ApJ, 596, 561

Murakawa, K., Suto, H., Tamura, M., et al. 2004, PASJ, 56, 509

Nakajima, T., Tsuji, T., \& Yanagisawa, K. 2004, ApJ, 607, 499

Neuhäuser, R., \& Brandner, W. 1998, A\&A, 330, L29

Neuhäuser, R., Sterzik, M. F., Schmitt, J. H. M. M., Wichmann, R., \& Krautter, J. 1995, A\&A, 297, 391

Neuhäuser, R., Brandner, W., Alves, J., Joergens, V., \& Comerón, F. 2002, A\&A, 384, 999

Reid, I. N., Kirkpatrick, J., Liebert, J., et al. 1999, ApJ, 521, 613

Reid, I. N., Burgasser, A. J., Cruz, K. L., Kirkpatrick, J. D., \& Gizis, J. E. 2001, AJ, 121, 1720

Rieke, G. H., \& Lebofski, M. J. 1985, ApJ, 288, 618

Rousset, G., Lacombe, F., Puget, P., et al. 2003, SPIE, 4839, 140

Schubert, G., \& Walterscheid, R. L. 2000, in Allen's Astrophysical Quantities, ed. A. N. Cox, AIP, 239

Stephens, D. C., Marley, M. S., Noll, K. S., \& Chanover, N. 2001, ApJ, 556, L97

Tachihara, K., Dobashi, K., Mizuno, A., Ogawa, H., \& Fukui, Y. 1996, PASJ, 48, 489

Teixeira, R., Ducourant, C., Sartori, M., et al. 2000, A\&A, 361, 1143

Tinney, C., Burgasser, A., \& Kirkpatrick, J. 2003, AJ, 126, 975

Wichmann, R., Bastian, U., Krautter, J., Jankovics, I., \& Rucinski, S. M. 1998, MNRAS, 301, L39

Wuchterl, G., Guillot, T., \& Lissauer, J. 2000, Protostars and Planets IV, ed. V. Mannings et al., 1081

Wuchterl, G., \& Tscharnuter, W. M. 2003, A\&A, 398, 1081 